\title{
The practicality of practice guidelines
}

$\mathrm{C}$ linical practice guidelines are often underused in Canada because developers fail to address potential barriers to implementation during the development and dissemination of recommendations, knowledge transfer experts told delegates to the Canadian Clinical Practice Guideline Summit in Gatineau, Quebec.

They're calling for a national strategy to standardize the development of clinical practice guidelines, a critical component of which would be a framework to help developers improve the "implementability" of recommendations.

Although the health care system is ultimately responsible for implementing best practices, guidelines developers "must make sure that handover is as smooth as possible," Jeremy Grimshaw, a senior scientist in clinical epidemiology at the Ottawa Hospital Research Institute in Ontario, told delegates. "The guidelines development process is a great opportunity to identify what the potential barriers are to the recommendations to help hospitals and primary care organizations think about the key issues they might need to address and the likely resource implications."

That includes simplifying the content of guidelines and disseminating recommendations in a variety of formats to better reflect and integrate with practitioner workflow, including nontraditional strategies such as embedding guidelines into electronic medical records as alerts or reminders.

"We should be tailoring interventions based upon diagnostic assessment of the barriers, understanding what's driving the behaviours of health care professionals," Grimshaw explained. "But quite often I see groups have a single intervention that they think will be effective in all circumstances. It's like someone having a hammer and thinking everything looks like a nail, when what we should have is a whole toolkit at our disposal and we should be able to know that a hammer is really good but what we need is a spanner."

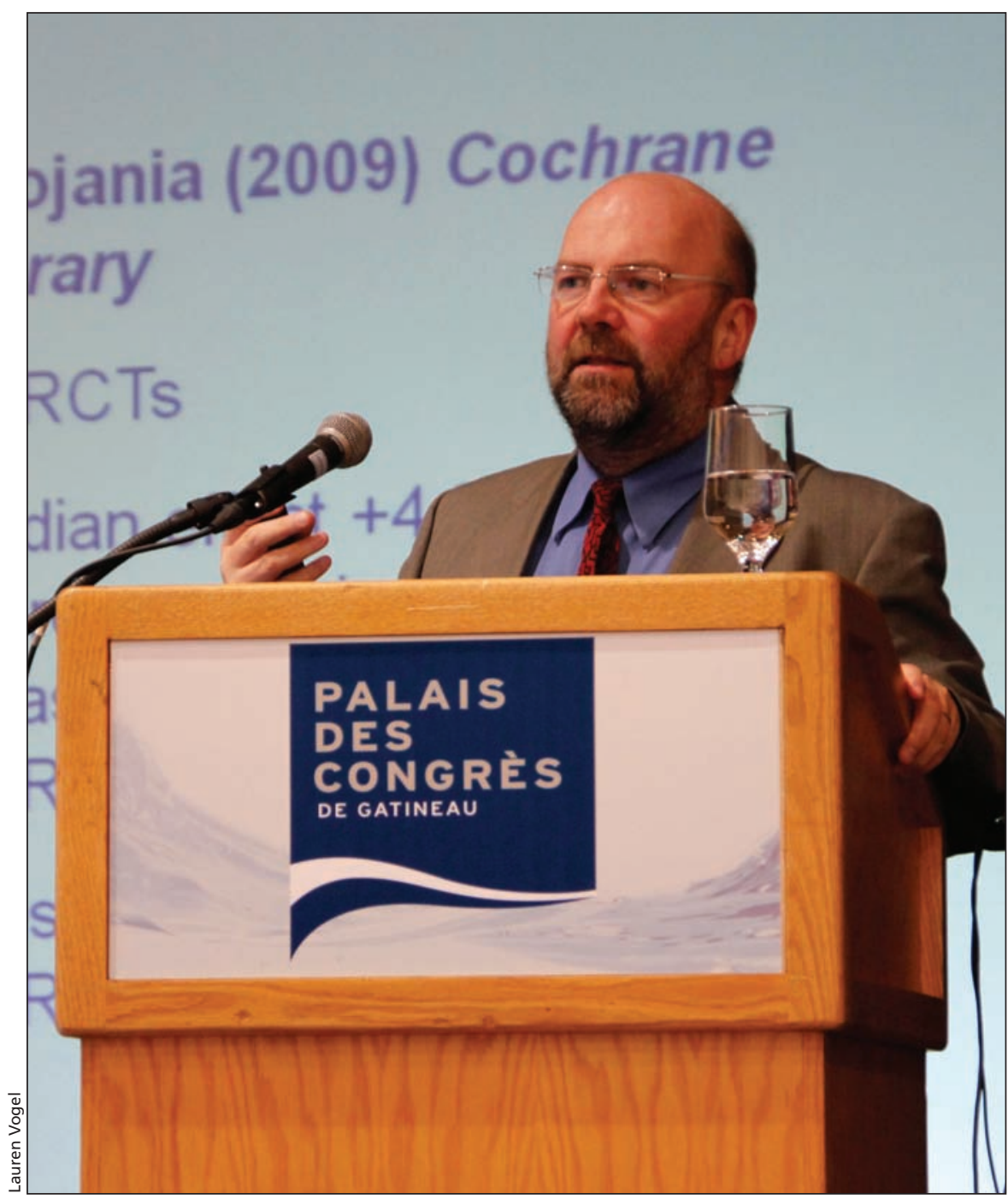

Guideline developers often "have a single intervention that they think will be effective in all circumstances," says Jeremy Grimshaw, a senior scientist in clinical epidemiology at the Ottawa Hospital Research Institute in Ontario. "It's like someone having a hammer and thinking everything looks like a nail, when what we should have is a whole toolkit at our disposal and we should be able to know that a hammer is really good but what we need is a spanner."

Development and implementation of clinical guidelines in Canada is fragmented and fraught with difficulty. With no central source to fund or coordinate guideline developers, many work in silos and rely heavily on industry funding (www.cmaj.ca/lookup/doi/10 .1503/cmaj.109-3784).

A national strategy could establish a process for developing evidence-based interventions to improve uptake of guidelines, such as conducting ongoing needs assessments during and after the development process to better understand how practitioners use the recommendations, said Carolyn Pullen, director of knowledge translation at the Canadian Cardiovascular Society.

Such an assessment could also look at the economic viability of recommendations, Grimshaw said. "Some professional groups would find that difficult. 
But I think it's a debate we need to have because it may well be we can develop guidelines based on efficiency that will get us $90 \%$ of the way there at $50 \%$ of the cost."

Limited time and resources are frequently among the top barriers to physician uptake of best practices, several delegates indicated.

The sheer number of guidelines produced, not to mention the length and complexity of the documents, often pose a major disincentive for health professionals to simply read, let alone employ, recommendations, said Patrice Lindsay, performance and standards specialist for the Canadian Stroke Network. "There has to be a common language and common ways to present the guidelines."

The rampant use of "weasel words" in guidelines, and lack of "easily understandable, actionable items" adds to the confusion, said Grimshaw. "We use just terrible language. You can imagine a physician or a nurse or a pharmacist looking at a guideline thinking they have no idea what it means. If we say the recommendations clearly, they're more likely to be used."

The "magic" is in the dissemination, agreed Pullen. "Journal publication is really just the first step. One of the best things we do for our biggest guidelines projects is we develop a workshop program where we'll insert ourselves in relevant national meetings and deliver the guidelines through interactive, casebased workshops."

Guidelines can also be delivered through e-learning programs, multimedia tools, such as podcasts, and smart phone applications, she added.

Implementing a measure of guideline standardization through a national strategy could also open new avenues for dissemination through embedding recommendations into electronic medical records, argued Maureen Charlebois, chief nursing executive and group director, clinical adoption, at Canada Health Infoway. "The future is having an integrated system to enable patient-centred care to ensure our clinicians have intelligent system to assist them in their care delivery for our patients."

Some software already integrates best practice recommendations into electronic health records as, for example, alerts that pop up when a physician orders tests that aren't indicated for the patient's condition, explained Dr. Martin Reed, chair of the Canadian Association of Radiologists' guidelines working group. "I get this guideline information saying what you're ordering is not necessary or appropriate, and then I can click to keep the current exam or I can reset the order." - Lauren Vogel, CMAJ

CMAJ 2011. DOI:10.1503/cmaj.109-4048 